\title{
Insulinoma in tuberous sclerosis
}

\author{
An entity not to be missed
}

Mohammed S. Al Qahtani, MD, EMSHA, Shoukat A. Bojal, MBBS, FRCS, Abdullah A. Alqarzaie, MBBS, Abdulaziz A. Alqabtani, MBBS.

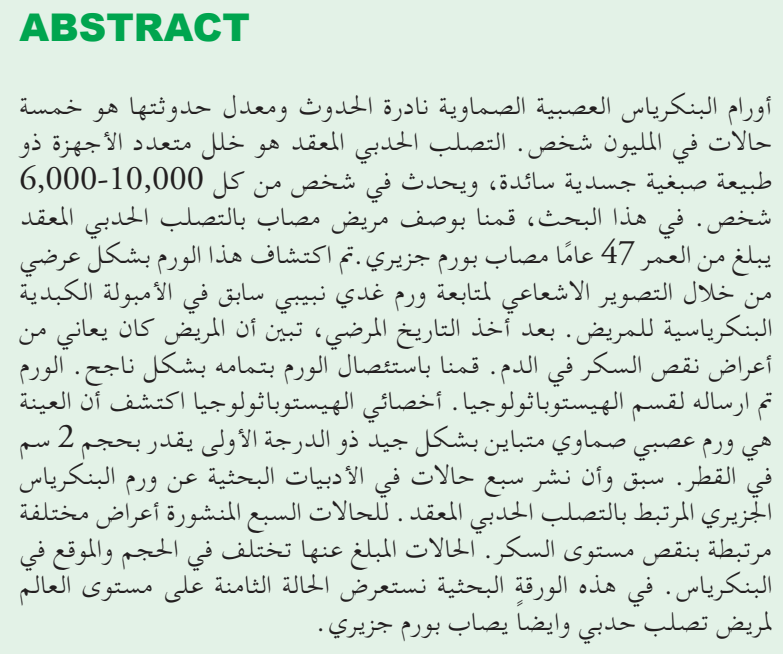

Pancreatic neuroendocrine tumors are rare with an incident rate of 5 cases per million individuals. Tuberous sclerosis complex is an autosomal dominant disease. This disease involves multisystem and occurs in one out of every 6,000-10,000 individuals. In this study, we describe a 47-year-old male known tuberous sclerosis patient with an insulinoma. The tumor was incidentally detected in follow-up imaging for a previous ampulla of Vater tubular adenoma. However, the patient reported symptoms of hypoglycemia. The insulinoma was enucleated successfully. Histopathology revealed a well-differentiated, grade one neuroendocrine tumor measuring around $2 \mathrm{~cm}$ in diameter. Seven cases were reported in the literature of tuberous sclerosisassociated insulinoma. The 7 reported cases had different hypoglycemia related symptoms. The reported tumors varied in size and location on the pancreas. This paper details the eighth case worldwide where an insulinoma occurred in a tuberous sclerosis patient.

Keywords: insulinoma, neuroendocrine tumors, tuberous sclerosis, case reports, review

Saudi Med J 2021; Vol. 42 (3): 332-337 doi: 10.15537/smj.2021.42.3.20200490
From the Department of Surgery (Al Qahtani M), Multi Organ Transplant Center; from the Department of Surgery (Bojal), King Fahad Specialist Hospital; and from the College of Medicine (Alqarzaie, Alqahtani A) Imam Abdulrahman Bin Faisal University, Dammam, Kingdom of Saudi Arabia.

Received 23rd September 2020. Accepted 10th December 2020.

Address correspondence and reprint request to: Dr. Mohammed S. Al Qahtani, Multi Organ Transplant Center King Fahad Specialist Hospital, Dammam, Kingdom of Saudi Arabia. E-mail:mohammedsaad.qahtani@kfsh.med.sa

ORCID ID: https://orcid.org/0000-0002-4122-4954

$\mathrm{P}$ ancreatic neuroendocrine tumors (NETs) are comparatively rare, considering their low incident rate of 5 cases per million individuals. ${ }^{1}$ Although most pancreatic NETs are non-functional, insulinomas are the most prevalent functional pancreatic NETs. ${ }^{1}$ While most pancreatic NETs are malignant, $90 \%$ of insulinomas are benign. ${ }^{1}$

Tuberous sclerosis complex (TSC) is an autosomal dominant disease, known to affect multisystem, and described by the growth of hamartomas. ${ }^{2}$ Two genes are involved in TSC pathogenesis: the TSC1 (9q34) which lead to hamartin and TSC2 (16q13.3) to tuberin coding. ${ }^{2}$ Tuberous sclerosis complex has an incidence of one case in every 6,000-10,000 individuals. ${ }^{2}$ It involves cellular migration, proliferation, and differentiation from a pathophysiological perspective, and affects several organs including the central nervous system, kidney, gastrointestinal tract, bones, and lungs. ${ }^{2}$

In this report, we present a very rare case where an insulinoma occurred in a TSC patient. As we performed a literature review, we found this case to be the eighth case as 7 cases were published in the literature. Moreover, previous reports have described the possible mechanisms of developing insulinoma in TSC patients. ${ }^{3,4}$ They described the role of the TSC2 gene in the growth of pancreatic islet cell tumors, and the mechanistic target of rapamycin (mTOR) pathway 
abnormality and insulinoma in TCS patients as possible mechanisms. ${ }^{3,4}$

Case Report. Patient information. This case concerns a 47-year-old man with TSC and epilepsy, who was on medications at the same time. In January 2008, he underwent brain computed tomography (CT), which revealed cortical tubers in the left frontal lobe and the suprasellar region. In the same month, he was diagnosed with renal epithelioid angiomyolipoma. $\mathrm{He}$ also had a history of familial adenomatous polyposis. Therefore, in July 2008, he underwent laparoscopic total proctocolectomy and ileoanal anastomosis with covering ileostomy. In 2009, he started to follow up for epilepsy in the neurology clinic at King Fahad Specialist Hospital (KFSH), Dammam, Saudi Arabia. Furthermore, he maintains seizures free for 10 years. On January 9, 2018, he underwent renal angioembolization for angiomyolipoma.

Clinical findings. On January 29, 2018, he started to develop epigastric pain. He underwent esophagogastroduodenoscopy and a biopsy of the ampulla of vater revealed tubular adenoma. In December 2018, a follow-up CT scan revealed an incidental pancreatic head lesion.

Diagnostic assessment. Further history taking from the patient revealed sweating and tremor as complaints symptoms of hypoglycemia. In the same month, December 2018, he was injected with $25 \mathrm{mCi}$ of Tc-99m tektrotyd (octreotide), and the pancreatic lesion was confirmed to be octreotide-avid in an octreotide scan (Figure 1).

Computed tomography enterography was conducted to rule out small bowel lesions. A CT scan revealed a stable enhancing lesion in the uncinate process of the pancreas. No definite hyper vascular focal lesion was detected in small bowel loops.

Therapeutic intervention. In March 2019, he underwent enucleation of the pancreatic uncinate tumor in the hepatobiliary section of KFSH. A midline laparotomy, followed by intraoperative ultrasound (IOUS), confirmed a mass located in the uncinate process of the pancreas. The mass was enucleated with no intraoperative or postoperative complications. The tumor was excised as a whole and appeared to be noninvasive on gross examination. It measured

Disclosure. Authors have no conflict of interests, and the work was not supported or funded by any drug company.
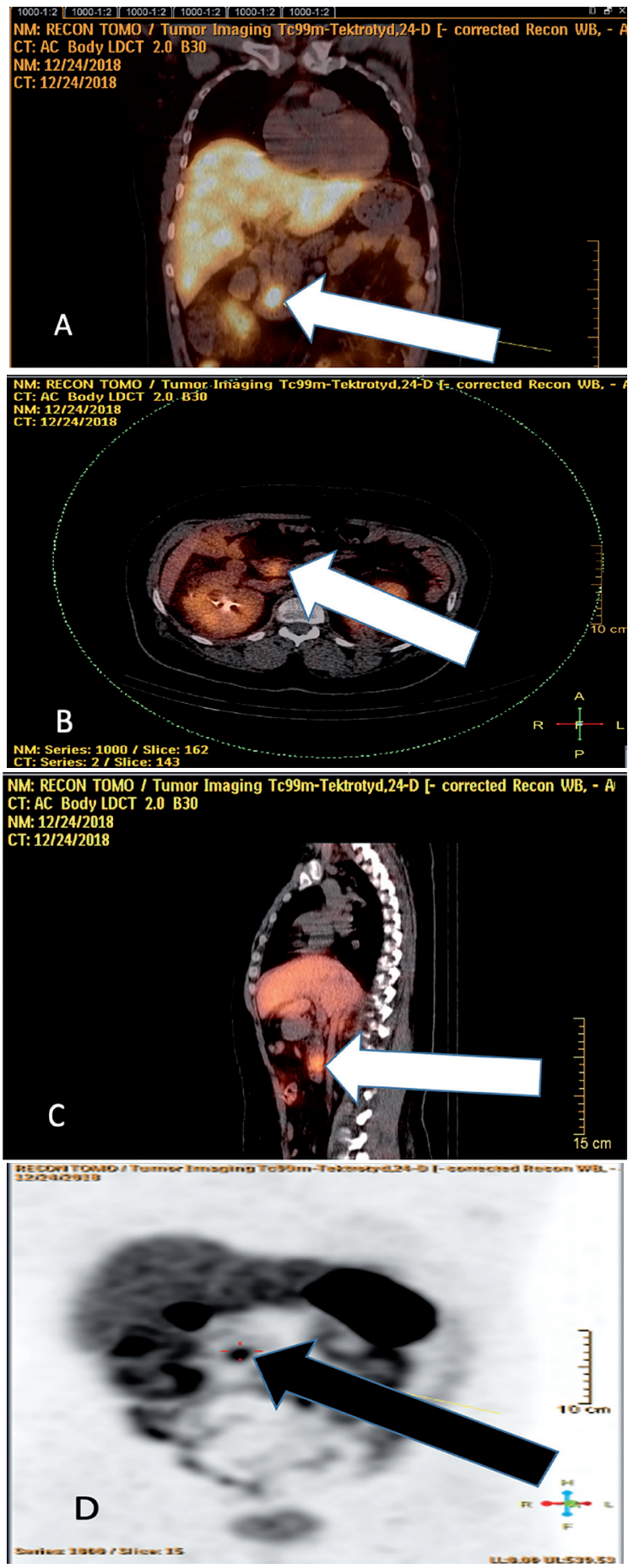

Figure 1 - The patient was injected with $25 \mathrm{mCi}$ of Tc-99m tektrotyd (octreotide) followed by scan of the whole body and static images and single photon emission computed tomography (SPECT)/CT for chest, abdomen, and pelvis. Above images show scintigraphic evidence of octreotide avid lesion in the pancreatic head A) coronal view, B) axial view, C) parasagittal SPECT/CT images. D) Shows maximum intensity projection (MIP) image, with arrow pointing to the head of pancreas, showing octreotide avid lesion. 
$2 \mathrm{~cm}$ in diameter, and was sent to the histopathology department for a frozen section analysis. Intraoperative ultrasound did not reveal other pancreatic or ductal lesions and the pancreatic duct integrity was confirmed post-procedure. The patient's timeline was summarized in Figure 2.

The histopathology of the frozen section reported a well-differentiated grade one NET has a mitotic activity of less than 2 per 10 high power fields. The Ki67 index was less than $2 \%$. Positive tumor cells for CD56 and synaptophysin (Figures 3A - 3D).

Follow-up and outcomes. The postoperative period and follow-up was uneventful. The hypoglycemic symptoms subsided after the surgery. Provided the histopathological findings, he remains on an oncological follow-up for surveillance, and is not receiving any active treatment. He is in regular follow up with hepato-pancreato-biliary (HBP) surgery clinic and he is in a stable condition. Verbal consent was obtained at the latest follow-up, which was documented in the patient file and prior to drafting this case report.

Discussion. We report the case of a 47 -year-old man with TSC and an insulinoma, which was successfully enucleated in March 2019. The occurrence of these 2 rare conditions in the same patient is extremely rare worldwide and is not coincidental, since very few reports have mentioned/described the correlation between the 2 conditions. ${ }^{2,3,5}$

Previous reports have described the relationship between tuberous sclerosis and malignant pancreatic islet cell tumor (a pancreatic NET) using deoxyribonucleic acid (DNA) analysis and immunohistochemistry. ${ }^{6}$ While DNA analysis revealed a loss of heterogenicity (LOH) on chromosome 16p13 in malignant pancreatic islet cells, no $\mathrm{LOH}$ was observed in the normal variant

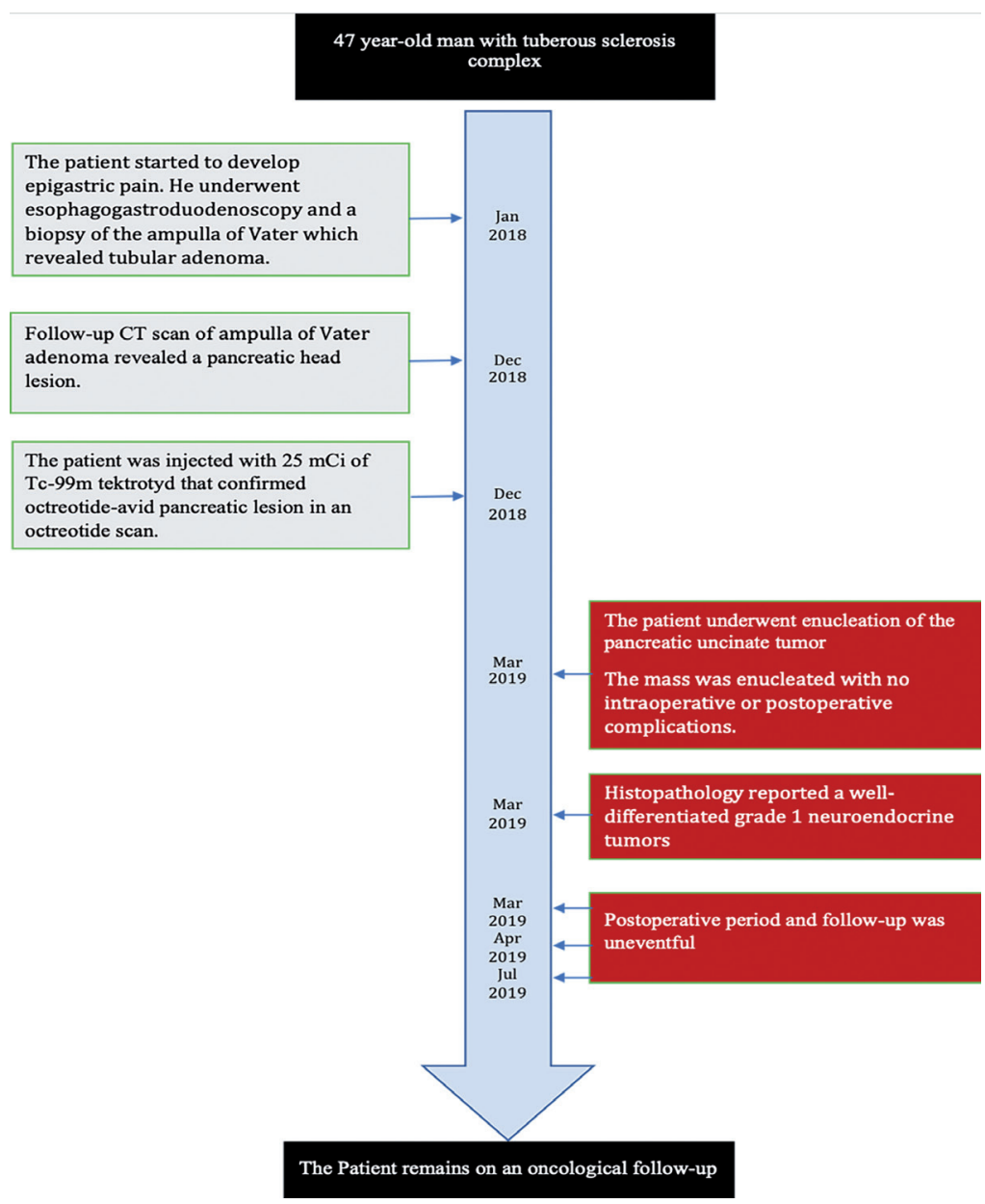

Figure 2 - Timeline flowchart of the patient with a diagnosis of tuberous sclerosis complex. 

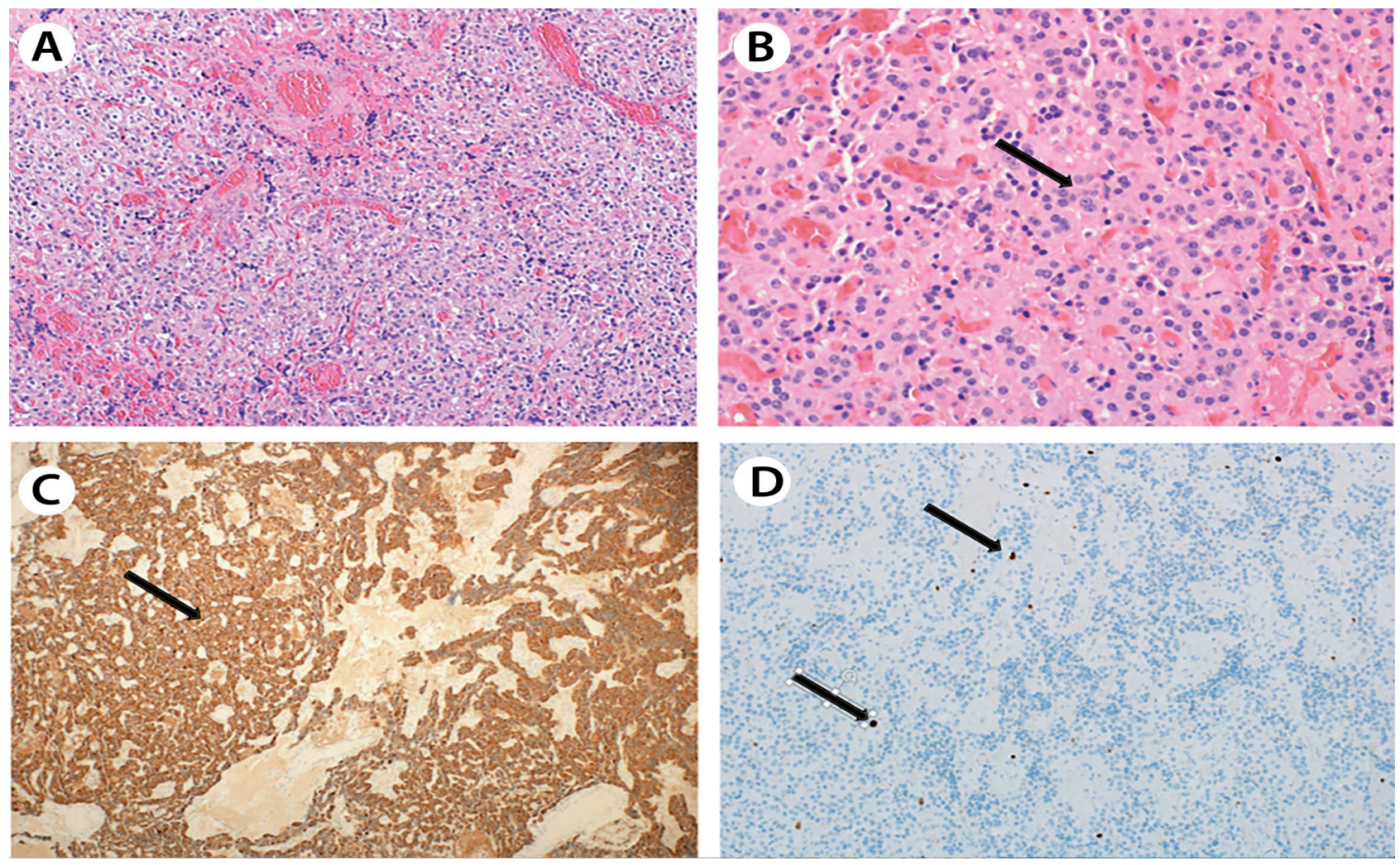

Figure 3 - A) Histological examination shows diffuse infiltration by bland-looking neoplastic epithelial cells arranged in cords, solid nests, and trabeculae $(\times 100$, hematoxylin and eosin). B) The neoplastic cells are small to medium sized showing low grade neuroendocrine features in the form of very rare mitotic figures, a salt-and-pepper chromatin pattern, uniform round nuclei, and a rim of amphophilic finely granular cytoplasm, (black arrow) ( $\times 200$, Hematoxylin\&Eosin). C) Diffusely positive neoplastic cells for neuroendocrine markers, chromogranin and synaptophysin (black arrow), (x100). D) Immunohistochemical stain for Ki67 highlights the very low mitotic activity of these neoplastic cells, less than $2 \%$ (black arrows), (x 200).

of these cells. ${ }^{6}$ Furthermore, immunohistochemical analysis using anti-tuberin antibodies resulted in a loss of tuberin immunostaining in the tumor cells; the rest of the pancreas were normal. ${ }^{6}$ These results suggested a role of the TSC2 gene product, tuberin, which is described in the pathogenesis of TSC, in the development of pancreatic islet cell tumors. ${ }^{6}$ A series of data suggested continuous activation of insulin receptor signaling due to a loss of tuberin in mutated TSC 2 alleles. This is supported by the observation that the tuberin-hamartin complex is essential in the regulation of the insulin receptor signal transduction. Thus, a mutation in the TSC2 plays a role in insulin regulation. ${ }^{6}$

On reviewing previous hypotheses that suggested a relationship between insulinomas in TSC patients and an mTOR pathway abnormality, mTOR was found to play a major role in the proliferation, growth, and survival of the cell. ${ }^{3}$ The activation of mTOR requires a series of events. ${ }^{3}$ The TSC1/ TSC2 (hamartin-tuberin) complex acts as a guanosine triphosphate pase protein
Table 1 - Literature review of insulinoma cases in tuberous sclerosis complex patients.

\begin{tabular}{lcccc}
\hline Demographics & \multicolumn{2}{c}{ Tumor characteristics } & References \\
Gender & $\begin{array}{c}\text { Age } \\
\text { (years) }\end{array}$ & $\begin{array}{c}\text { Size } \\
(\mathrm{cm})\end{array}$ & $\begin{array}{c}\text { Site in the } \\
\text { pancreas }\end{array}$ & \\
\hline $\mathrm{F}$ & 24 & 3 & Body & $(10)$ \\
$\mathrm{M}$ & 23 & 3 & Head & $(11)$ \\
$\mathrm{M}$ & 28 & 2 & Tail & $(12)$ \\
$\mathrm{M}$ & 18 & 2.9 & Head & $(13)$ \\
$\mathrm{M}$ & 43 & 21 & $\begin{array}{c}\text { Not clearly } \\
\text { mentioned }\end{array}$ & $(8)$ \\
$\mathrm{M}$ & 23 & $\begin{array}{c}\text { Tumor } 1: 3.5 \\
\text { Tumor 2: } 0.7\end{array}$ & Tail & $(3)$ \\
$\mathrm{F}$ & 67 & 1.5 & Uncinate process & $(5)$ \\
\hline
\end{tabular}


that negatively regulates the mTOR using the Ras homolog enriched in the brain, a direct positive regulator. ${ }^{3}$ In order to enhance cell growth, this TSC1/ TSC2 complex needs to be inhibited. ${ }^{4}$

The insulin-like growth factor-1, a stimulant, bind to the tyrosine kinase receptor and induce phosphorylation of the phosphoinositide 3-kinase (PI3K). ${ }^{4}$ This initiates a phosphorylation cascade, which activates the protein kinase B. ${ }^{4}$ Protein kinase B then inhibits the TSC1/ TSC2 complex, resulting in cell growth and proliferation. ${ }^{4}$ Insulin signaling is controlled by the mTOR pathway. ${ }^{7}$ Thus, TSC1 or TSC2 mutations would lead to the constitutive activation of the mTOR and an abnormal regulation of insulin signaling. ${ }^{7}$

In 2009, a large systematic review of NET in TSC patients, reported between 1950 and 2008 in all languages in the PubMed database. ${ }^{2}$ It discovered 5 cases of insulinomas in TSC patients, the first case was published in $1959 .^{2}$ Subsequently, 2 cases were published in $2017^{3}$ and $2018^{5}$ each. As of today, a total of 7 cases, 4 men and 3 women have been reported worldwide. ${ }^{3,4}$ The youngest case was of an 18-year-old woman, which was reported in 1997 , while the oldest case was of a 67-year-old woman, which was reported in 2018. ${ }^{2,4}$ Our case, the eighth in the world is of a 47-year-old man.

The median age of developing a sporadic insulinoma was reported as 50 years. ${ }^{3}$ Conversely, cases with TSCassociated insulinomas were reported to have a median age of 26 years (range: 18 to 67 years, with the median age of 5 of the 8 cases being 28 years). ${ }^{3,4}$ In a study conducted in 132 insulinoma patients by the Mayo Clinic, the mean tumor size was found to be $1.5 \mathrm{~cm}$, with the largest tumor measuring $4 \mathrm{~cm}^{8}$ However, TSC-associated insulinomas were found to vary in size between $7 \mathrm{~mm}$ (the smallest) and $21 \mathrm{~cm}$ (the largest ever reported). ${ }^{3,8}$ There are variations in the sites of TSC-associated insulinomas. ${ }^{3,4}$ Table 1 summarizes all the published cases.

While all 7 cases presented initially with hypoglycemic symptoms (which raised the suspicion of an insulinoma), our case was detected during routine follow-up. ${ }^{3,4}$ Appropriate history taking by the treating team discovered hypoglycemic complaints by the patient. After the surgery, the histological diagnosis was a well-differentiated pancreatic neuroendocrine tumor, World Health Organization grade one. This finding correlates clinically with insulinoma based on patient clinical symptoms.

Surgical interventions include laparoscopic and open enucleation, or more extensive procedures such as distal pancreatectomy or pancreaticoduodenectomy. The surgical choice in our patient was guided by his history and the pre-operative imaging results. Surgical enucleation was assisted by IOUS for confirming the lesion and its proximity to the pancreatic duct, in order to prevent pancreatic duct injury. The management of insulinoma depends on many factors. Surgical management is the first line of treatment with a $77 \%-100 \%$ cure rate; however, medical management is reserved for those unfit for surgery or with symptomatic metastasized tumors. ${ }^{?}$

While enucleation is the treatment of choice, other procedures (laparoscopic or open), such as the segmental resection of the pancreas, distal pancreatectomy, or pancreaticoduodenectomy (Whipple procedure), are undertaken for insulinomas that are not amenable for enucleation due to anatomical reasons. ${ }^{9}$ The lesions that are located in the body and tail of the pancreas, or in the head of the pancreas which are closely related to the pancreatic duct; these are managed by different surgical options. In these patients, provided the higher risk of complications (such as a pancreatic duct injury or fistula formation), enucleation may not be preferable. In our patient, the lesion was located in the uncinate process of the pancreas and was successfully enucleated without any postoperative complications.

Medical or surgical management and the type of surgical procedure depends on the preoperative assessment of possible metastasis and localization. ${ }^{9}$ In our patient, CT enterography excluded metastasis. Kang et $\mathrm{al}^{3}$ observed that a preoperative multiphase CT scan failed to detect a small 7-mm lesion. ${ }^{3}$ In our case, we employed IOUS, which ruled out pancreatic and ductal lesions, a dilated pancreatic duct, or any other injury.

Patient perspective. The finding of the pancreatic tumor made the patient concerned on the tumor and the need for further investigation and surgery. Appropriate assurance by the treating team to the patient and his family eased their concerns. After the surgery, the team informed the patient on how the tumor was excised as a whole. The patient was relieved. Postoperatively the patient was doing well and tolerated the procedure, also was satisfied with the management pathway. The patient is on a regular follow-up plan with the oncology clinic and HBP clinic. Moreover, he was informed of writing a case report on his condition and how this will add benefit to the literature.

In conclusion, few cases of TSC-associated insulinomas have been described in the literature. The pathogenesis of TSC-associated insulinomas is strongly 
connected with an abnormal mTOR pathway and is especially correlated with TSC2 (tuberin) inhibition.

Our case was found initially by imaging then we discovered hypoglycemic symptoms, we recommend screening for hypoglycemia and detailed investigations for insulinomas in all patients with TSC. Further studies are needed to explain the relationship between TSC and insulinomas.

Acknowledgment. We would like to thank Dr. Amani A. Joudeh, Surgical Pathologist Consultant, King Fahad Specialist Hospital, Dammam, Saudi Arabia for assisting us in obtaining pathology slides and writing the description for the insulinoma biopsy. Also, we would like to thank Dr. Abdulrhman Al Naim, Head of Nuclear Medicine, King Fahad Specialist Hospital, Dammam, Saudi Arabia for assisting us in reviewing and writing the description for the radiology pictures. Also, we would like to thank Editage (www.editage.com) for the English language editing.

\section{References}

1. Brunicardi FC, Andersen DK, Billiar TR, Dunn DL, Kao LS. Hunter JG, et al. Schwartz's principles of surgery, 11th ed. In: Moyer A, Naglieri C, Elsbury K. Garima Poddar, editors. New York: McGraw-Hill; 2019.

2. Dworakowska D, Grossman AB. Are neuroendocrine tumours a feature of tuberous sclerosis? A systematic review. Endocr Relat Cancer 2009; 16: 45-58.

3. Kang MY, Yeoh J, Pondicherry A, Rahman H, Dissanayake A. Insulinoma and tuberous sclerosis: a possible mechanistic target of rapamycin (mTOR) Pathway Abnormality? J Endocr Soc 2017; 1: 1120-1123.
4. Orlova KA, Crino PB. The tuberous sclerosis complex. Ann N Y Acad Sci 2010; 1184: 87-105.

5. Comninos AN, Yang L, Abbara A, Dhillo WS, Bassett JH, Todd JF. Frequent falls and confusion: recurrent hypoglycemia in a patient with tuberous sclerosis complex. Clin Case Rep 2018; 6: 904-909.

6. Francalanci P, Diomedi-Camassei F, Purificato C, Santorelli FM, Giannotti A, Dominici C, et al. Malignant pancreatic endocrine tumor in a child with tuberous sclerosis. Am J Surg Pathol 2003; 27: 1386-1389.

7. Khamzina L, Veilleux A, Bergeron S, Marette A. Increased activation of the mammalian target of rapamycin pathway in liver and skeletal muscle of obese rats: possible involvement in obesity-linked insulin resistance. Endocrinology 2005; 146 : 1473-1481.

8. Eledrisi MS, Stuart CA, Alshanti M. Insulinoma in a patient with tuberous sclerosis: is there an association? Endocr Pract 2002; 8: 109-112.

9. Shin JJ, Gorden P, Libutti SK. Insulinoma: pathophysiology, localization and management. Future Oncol 2010; 6: 229-237.

10. Gutman A, Leffkowitz M. Tuberous sclerosis associated with spontaneous hypoglycaemia. BMJ 1959; 2: 1065-1068.

11. Davoren PM, Epstein MT. Insulinoma complicating tuberous sclerosis. J Neurol Neurosurg Psychiatry 1992; 55: 1209.

12. Kim H, Kerr A, Morehouse H. The association between tuberous sclerosis and insulinoma. Am J Neuroradiol 1995; 16 : 1543-1544.

13. Boubaddi NE, Imbert Y, Tissot B, Chapus JJ, Dupont E, Gallouin D, et al. Secreting insulinoma and Bourneville's tuberous sclerosis. Gastroenterol Clin Biol 1997; 21: 343. 